\title{
Sistemas de representación y aprendizaje de las matemáticas
}

\author{
Pedro Javier Rojas G. \\ pedroedumat@udistrital.edu.co \\ Universidad Distrital Francisco José de Caldas \\ Grupo MESCUD
}

\subsection{Introducción}

En el contexto de la lengua castellana, la Real Academia Española (RAE) define representar como "Hacer presente algo con palabras o figuras que la imaginación retiene" y representación en tanto acción y efecto de representar" como "Figura, imagen o idea que sustituye a la realidad"; en otras palabras, "mostrar una cosa en cambio de otra' ${ }^{2}$ En tal sentido, se debe tener presente que es diferente la representación de un objeto (ya sea concreto o abstracto) y el objeto propiamente dicho; una cosa es "Juan" como sujeto concreto (perceptible directamente) y otra una fotografía, una caricatura o una descripción verbal (oral o escrita) que se haga de él; una cosa es el concepto de "Estado" y otra cada una de las posibles representaciones que de este concepto se pueda tener ${ }^{3}$

\subsection{Representación en matemáticas.}

En este escrito, y sin establecer en un primer momento diferencias con la definición de representación antes referida, se abordará de una manera general lo relativo a la representación en matemáticas y el uso de una diversidad de tales representaciones. Sin embargo, es importante aclarar que en las distintas acepciones de representar, que se encuentran en el diccionario de la RAE, ninguna de ellas hace referencia a la posibilidad de existencia de representaciones no conscientes, ni de representaciones que no estén en lugar de un objeto, como es el caso de las representaciones mentales inconscientes y computacionales 4 sobre las cuales se hará referencia posteriormente.

En matemáticas, el aprendizaje de los objetos es conceptua 5 , el sujeto no entra en ?contacto? directo con un determinado objeto, sino con una(s) representación(es) particular(es) de este objeto matemático. Incluso,

\footnotetext{
${ }^{1}$ Tomado de la vigésima segunda edición del Diccionario de la Lengua Española (RAE, 2001).

${ }^{2}$ Aquí se hace referencia a representaciones externas, o mejor, a representaciones ostensivas en cuanto pueden ser mostradas a otros. Por otra parte, si bien podría realizarse una discusión en torno a lo que se considera como realidad, y si ésta existe independiente del sujeto, no es el interés de este breve escrito.

${ }^{3}$ Ver, por ejemplo, D’Amore B. (2005). Pipas, caballos, triángulos y significados. Contribuciones a una teoría problemática del significado conceptual, desde Frege y Magritte, hasta nuestros días. Versión digital disponible en: http://www.dm. unibo.it/rsddm/ it/articoli/damore/damore.htm

${ }^{4}$ Tienen carácter algorítmico, su función es de tratamiento de una serie de datos en otra serie de datos, y no de evocación de objetos, ni de hacerlos visibles.

${ }^{5}$ Según lo plantea Duval, la adquisición conceptual de un objeto pasa por adquirir representaciones semióticas, esto es, representaciones por medio de signos.
} 
desde el realismo ingenuo, podría afirmarse que todo concepto matemático remite a un no-objeto y por tanto no son posibles las referencias ostensivas y hay una obligatoriedad a servirse de representaciones. Por ejemplo, el número seis suele ser representado con el signo “6”, pero este signo, esta marca, no es el número seis, tan sólo es una de las diversas maneras de representarlo. Podría escogerse otros signos, como:

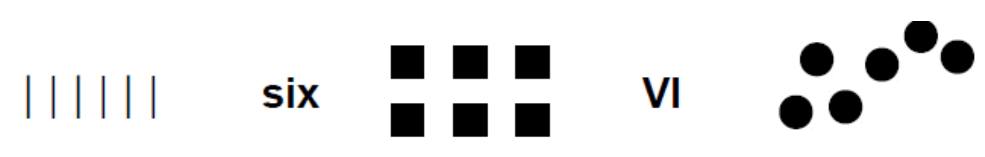

Ahora bien, las representaciones de un objeto que en un momento dado se escogen no son neutras, en tanto diferentes representaciones pueden "destacar" distintas características o elementos diferenciadores del objeto que pretenden representar; o mejor, diferentes "unidades significantes". Por ejemplo, en una caricatura de Juan se puede resaltar rasgos de él que posiblemente no son tan evidentes en una fotografía; en otro caso, una determinada configuración de "puntos", como la primera de las dos propuestas anteriormente para representar el número seis, puede "sugerir" descomposiciones casi inmediatas de este número, como $2+2+2$ o $3+3$, que no parece tan clara en la segunda; aunque si se tratara de realizar operaciones con estos signos, posiblemente ninguna de estas configuraciones tendría la utilidad del signo " 6 ", usualmente llamado numera 6 ,

Desde lo planteado por Duval ([3]), existen tres polos constitutivos de la representación que no deben confundirse:

a) el objeto representado

b) el contenido de la representación, es decir, lo que una representación particular presenta del objeto

c) la "forma" de la representación, es decir, su modalidad o su registro.

Este autor destaca que las representaciones pueden depender de sistemas semióticos (sistemas de signos), o de sistemas no semióticos (como en el caso de las redes neuronales o los instrumentos físicos). Insiste Duval en que:

"una representación jamás puede ser considerada y analizada sin hacer referencia al sistema a través del cual fue producida. Las especificidades del sistema (físico, orgánico o semiótico) que permitieron la producción de una representación, son las que determinan la relación entre el contenido y el objeto representado. El contenido de las representaciones de un mismo objeto cambia en función del sistema por el cual fueron producidas" (págs 18-19).

Si bien para efectos de la comunicación, de tipo general, puede ser suficiente el uso de un sistema de representación, como el lenguaje natural (oral o escrito) -sin negar la utilidad que pueda tener el usar otro sistema de representación (icónico, gráfico, tabular, etc.) para destacar propiedades diferentes de un mismo objeto-; para el caso de las matemáticas, Duval (pág 18) reconoce que el uso de más de un sistema de representación y la posibilidad de realizar transformaciones entre los diferentes sistemas resulta ser "una exigencia cognitiva necesaria y fundamental"; más aún, plantea explícitamente que "estos sistemas [semióticos...] son tan necesarios para el desarrollo del pensamiento matemático como la innovación y el perfeccionamiento de instrumentos de óptica o de medida en otras disciplinas científicas. La mediación semiótica es tan indispensable en matemáticas como la mediación instrumental para la observación de los fenómenos' 7 . Profundizando en las ideas anteriormente dichas, puede concluirse que para el aprendizaje de las matemáticas el uso, el estudio y la comprensión de los sistemas de representación son imprescindibles 8 , entre otras razones, porque:

\footnotetext{
6?'Qué podría sustentar la plausibiliadad de esta afirmación?

${ }^{7}$ Desde lo planteado por Luis Moreno Armella "Toda acción cognitiva es una acción mediada por instrumentos materiales o simbólicos" [Notas del curso de Didáctica de las Matemáticas, Cali, 1995].

${ }^{8}$ Varias investigaciones en Didáctica de las Matemáticas, dan cuenta de las dificultades que encuentran los niños y jóvenes al ser "inducidos" por la enseñanza a asociar los objetos matemáticos no sólo con un sistema de representación sino, más grave aún, con una única representación en este sistema, como es el caso del triángulo reducido a una imagen de triángulo equilátero que "descansa" sobre uno de sus lados. Ver, por ejemplo, D’Amore (2006, p. 137-142).
} 
a) Los objetos matemáticos están dispuestos en una gran variedad de registros.

b) La naturaleza de los objetos matemáticos hace que la manera de acceder a ellos sea vía la representación.

c) La representación en un sistema hace "visible" unas características del objeto y no otrał: así que, entre más sistemas de representación "coordinados" tenga un sujeto, su conocimiento del objeto matemático será más potente y más complejo.

\subsection{Registros de representación.}

Según lo plantea Duval (pág. 43), desde lo propuesto por De Saussure, "un signo puede significar algo solo gracias a las relaciones de oposición que puede tener con otros signos. Un signo es tal, solo al interior de un conjunto de otros signos; su sentido está ligado a un valor de elección en relación con otros posibles". Para el caso de matemáticas, siguiendo las ideas de Duval, algunas de las notaciones utilizadas provienen de un sistema (como en el caso de las cifras en notación posicional de base $n$ ) y otras no (como en el caso de las letras como variables, donde cada vez debe explicitarse qué conjunto de números puede representar), en tanto son netamente convencionales.

Según lo planteado anteriormente, parece clara la necesidad de trabajar con sistemas de representación, o, más específicamente, registros como los denomina Duval ${ }^{10}$ En matemáticas, los registros pueden ser discursivos (usando una lengua natural -que constituye el sistema semiótico por excelencia-, o una lengua formal), por ejemplo, para dar una definición, para enunciar una proposición o para realizar cálculos algebraicos; o registros no discursivos, como las figuras geométricas o sistemas de coordenadas ${ }^{11}$

Ahora bien, el sujeto puede realizar transformaciones entre diferentes representaciones semióticas; estas pueden de dos tipos: tratamiento o conversión. Si la transformación se realiza al interior de un mismo registro semióticd ${ }^{12}$ se denomina transformación de tratamiento; por ejemplo, dos formas diferentes de formular una proposición, ambas en un lenguaje natural; o la realización de un cálculo algebraico, como $n(n+1)=n^{2}+n$. Si la transformación se realiza entre dos registros semióticos diferentes, se denomina transformación de conversión; por ejemplo, transformar una relación descrita en lenguaje natural, como " $n$ es un número positivo", en una relación expresada en lenguaje algebraico, como " $n>0$ ".

El siguiente ejemplo, basado en D’Amore ([1, pág. 17]), puede ilustrar aún más lo planteado en el párrafo anterior:

\footnotetext{
${ }^{9}$ Varias investigaciones en Didáctica de las Matemáticas, dan cuenta de las dificultades que encuentran los niños y jóvenes al ser "inducidos" por la enseñanza a asociar los objetos matemáticos no sólo con un sistema de representación sino, más grave aún, con una única representación en este sistema, como es el caso del triángulo reducido a una imagen de triángulo equilátero que "descansa" sobre uno de sus lados. Ver, por ejemplo, D $\Delta$ Amore (p. 137-142).

${ }^{10}$ Para este autor, no todo sistema de representación semiótica es un registro de representación semiótica. Para ser registro, incluye como requerimiento la posibilidad de realizar transformaciones entre representaciones semióticas. Así, por ejemplo, el sistema de señales de tránsito es un sistema de representación semiótica aunque no sería un registro de representación semiótica (una señal de tránsito no puede verse como equivalente o "descomponerse" en otras señales del sistema).

${ }^{11}$ Duval diferencia claramente entre registros multifuncionales (donde los tratamientos no son algoritmizables), asociados a representaciones discursivas o no, y registros monofuncionales (donde los tratamientos son principalmente algoritmizables) también asociadas a si son o no discursivas (pág. 52).

${ }^{12}$ Por ejemplo, en el lenguaje natural, en el sistema de numeración posicional, en el gráfico, en el tabular, en el sistema de escritura algebraica, entre otros.
} 
- Registro semiótico 1. Lenguaje natural

- Representación A: La mitad

- Representación B: Uno de dos

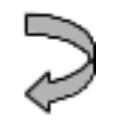

(Transformación de )

- Representación C: Un medio

- Registro semiótico 2. Lenguaje aritmético:

- Representación A: 1/2 (fraccionaria)

- Representación B: 0,50 (decimal)

- Representación C: $5 \cdot 10^{-1}$ (exponencial)

- Registro semiótico 3: Lenguaje algebraico

- Representación A: $\left\{x \in \mathbb{Q}^{+} \mid 2 x-1=0\right\}$

- Representación B: $y=f(x): x \longrightarrow x / 2$

- Registro semiótico 4. Lenguaje gráfico o esquema pictográfico
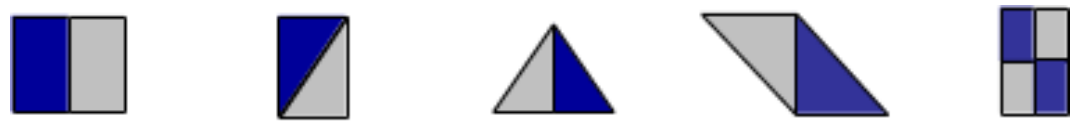

- Registro semiótico 5. Lenguaje figural

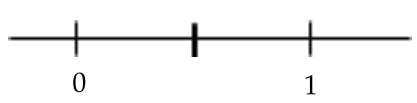

En síntesis, la construcción del conocimiento matemático requiere de tres acciones básicas, a saber:

a) Representar.

b) Tratar las representaciones al interior de un registro.

c) Convertir las representaciones de un registro a otro.

No obstante lo planteado hasta aquí, desde la propuesta de Duval, no puede concluirse que los problemas del aprendizaje y de la enseñanza de las matemáticas están resueltos, posiblemente esto aún esté muy lejos de suceder, entre otras razones, porque la formulación hasta aquí presentada lleva, al parecer, a una "paradoja cognitiva", la cual fue planteada por el mismo autor ([1, pág. 28]):

\footnotetext{
“¿Cómo puede aprender un estudiante a no confundir un objeto matemático con la representación particular que le da acceso (por ejemplo, un número y su escritura, una figura y la situación representada, un grafo y la función,...), si para acceder a los objetos representados no hay más que representaciones semióticas para manipular?"
}

Por otra parte, en un trabajo reciente, Rojas ([5, basado en ideas de D'Amore]), ha puesto en evidencia que las transformaciones de tratamiento pueden ser tan complejas como las de conversión y, por tanto, ser fuente de dificultades en los procesos de comprensión de la matemática escolar. A manera de ejemplo, plantea que si bien muchos estudiantes de último grado de educación básica y media (15-17 años) reconocen que la expresión algebraica $(n-1)+n+(n+1)$, que representa o puede ser interpretada como la suma de tres números enteros consecutivos, y la expresión $3 n$, que representa o puede ser interpretada como el triple de un número entero, son expresiones equivalentes en tanto pueden usar propiedades del sistema de los números enteros para probar que se cumple la igualdad, es decir, que $(n-1)+n+(n+1)=3 n$, para todo número entero $n$. 
Sin embargo, lo anterior no garantiza que reconozcan, por ejemplo, que la expresión $(n-1)+n+(n+1)$ pueda ser interpretada como el triple de un número, en tanto dicha equivalencia sintáctica de las expresiones no siempre posibilita que los estudiantes puedan "articular" las interpretaciones o significados asignados a cada una de las anteriores expresiones y puedan reconocer una equivalencia semántica.

Sin duda la problemática asociada con el significado de los objetos matemáticos, los procesos de representación y los procesos de aprendizaje, continúan siendo una temática importante en relación con el aprendizaje de la matemática escolar.

\section{Bibliografía}

[1] D’Amore, B. (2004). Conceptualización, registros de representaciones semióticas y noética: interacciones constructivistas en el aprendizaje de los conceptos matemáticos e hipótesis sobre algunos factores que inhiben la devolución. Uno, 35, 90-106.

[2] D’Amore B. (2006). Didáctica de la Matemática. Bogotá: Magisterio [Á. Balderas, Trad. versión original publicada en italiano por Pitagora, 1999].

[3] Duval R. (2001). Los problemas fundamentales en el aprendizaje de las matemáticas y las formas superiores en el desarrollo cognitivo. Cali: Universidad del Valle [M. Vega, Trad., notas originales en francés, Universidad del Valle, Colombia, 1999].

[4] Kaput, J. (1992). Technology and Mathematics Educations. In: Grouws, D. (Ed.). Handbook of Research Mathematics Teaching and Learning. New York: Macmillan, 515-556.

[5] Rojas (2009). Relación entre objeto matemático y sentidos en situaciones de transformación entre representaciones semióticas. Proyecto de tesis doctoral (no publicado), dirigido por el Dr. Bruno D?Amore. Bogotá, Universidad Distrital Francisco José de Caldas. 